\title{
Correction to: Introduction to Spherical Elementary Current Systems
}

Heikki Vanhamäki and Liisa Juusola

\section{Correction to:}

Chapter 2 in: M. W. Dunlop and H. Lühr (eds.),

Ionospheric Multi-Spacecraft Analysis Tools, ISSI Scientific Report Series 17, https://doi.org/10.1007/978-3-030-26732-2_2

The original version of this chapter was published without the Electronic Supplementary Material. It has now been included in Chapter 2. The erratum chapter has been updated with the change. 